\title{
Local and regional temporal trends (2013- 2019) of canine Ehrlichia spp. seroprevalence in the USA
}

Jenna R. Gettings ${ }^{1 *}$ (D) Stella C. W. Self², Christopher S. McMahan³ ${ }^{3}$ D. Andrew Brown ${ }^{3}$, Shila K. Nordone ${ }^{4}$ and Michael J. Yabsley ${ }^{1,5}$

\begin{abstract}
Background: In the USA, there are several Ehrlichia spp. of concern including Ehrlichia canis, Ehrlichia ewingii, Ehrlichia chaffeensis, Ehrlichia muris eauclarensis, and "Panola Mountain Ehrlichia". Of these, E. canis is considered the most clinically relevant for domestic dogs, with infection capable of causing acute, subclinical, and chronic stages of disease. Changes in climate, land use, habitats, and wildlife reservoir populations, and increasing contact between both human and dog populations with natural areas have resulted in the increased risk of vector-borne disease throughout the world.

Methods: A Bayesian spatio-temporal binomial regression model was applied to serological test results collected from veterinarians throughout the contiguous USA between January 2013 and November 2019. The model was used to quantify both regional and local temporal trends of canine Ehrlichia spp. seroprevalence and identify areas that experienced significant increases in seroprevalence.

Results: Regionally, increasing seroprevalence occurred within several states throughout the central and southeastern states, including Missouri, Arkansas, Mississippi, Alabama, Virginia, North Carolina, Georgia and Texas. The underlying local trends revealed increasing seroprevalence at a finer scale. Clusters of locally increasing seroprevalence were seen from the western Appalachian region into the southern Midwest, along the Atlantic coast in New England, parts of Florida, Illinois, Wisconsin and Minnesota, and in a couple areas of the Mountain region. Clusters of locally decreasing seroprevalence were seen throughout the USA including New York and the mid-Atlantic states, Texas, the Midwest, and California.

Conclusions: Canine Ehrlichia spp. seroprevalence is increasing in both endemic and non-endemic areas of the USA. The findings from this study indicate that dogs across a wide area of the USA are at risk of exposure and these results should provide veterinarians and pet owners with the information they need to make informed decisions about prevention of tick exposure.
\end{abstract}

Keywords: Ehrlichia canis, Ehrlichia chaffeensis, Ehrlichia ewingii, Temporal trends, USA, Vector-borne

*Correspondence: jrgettings@gmail.com

${ }^{1}$ Southeastern Cooperative Wildlife Disease Study, University of Georgia, Athens 30602, USA

Full list of author information is available at the end of the article

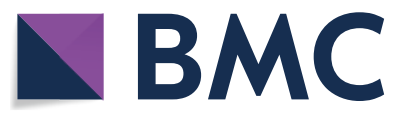

(c) The Author(s) 2020. This article is licensed under a Creative Commons Attribution 4.0 International License, which permits use, sharing, adaptation, distribution and reproduction in any medium or format, as long as you give appropriate credit to the original author(s) and the source, provide a link to the Creative Commons licence, and indicate if changes were made. The images or other third party material in this article are included in the article's Creative Commons licence, unless indicated otherwise in a credit line to the material. If material is not included in the article's Creative Commons licence and your intended use is not permitted by statutory regulation or exceeds the permitted use, you will need to obtain permission directly from the copyright holder. To view a copy of this licence, visit http://creativeco mmons.org/licenses/by/4.0/. The Creative Commons Public Domain Dedication waiver (http://creativecommons.org/publicdomain/ zero/1.0/) applies to the data made available in this article, unless otherwise stated in a credit line to the data. 


\section{Background}

The predominately recognized agents for canine ehrlichiosis in the USA include Ehrlichia canis, Ehrlichia ewingii and Ehrlichia chaffeensis [1]. Despite their shared genus, they are transmitted by different vectors: Amblyomma americanum is the primary vector of E. ewingii [2] and $E$. chaffeensis [3], and Rhipicephalus sanguineus is the primary vector of $E$. canis [4]. The distribution of infected dogs follows that of the associated tick vectors, and the seroprevalence of antibodies against $E$. ewingii or $E$. chaffeensis generally exceeds that of $E$. canis $[5,6]$. Recent evidence suggests that dogs are also susceptible to infection by Ehrlichia muris, believed to be transmitted by Ixodes scapularis [7] and "Panola Mountain Ehrlichia", which is transmitted by $A$. americanum [8]. In 2019, over 200,000 dogs tested positive for antibodies against Ehrlichia spp. within the USA out of 7,056,709 tested [9]. In Canada, over 1000 dogs tested positive out of 168,216 tested, but this is likely an underestimate as these data have just recently started being collected [9].

Several of these Ehrlichia spp. also infect people and the incidence of human ehrlichiosis, similar to other vector-borne diseases, has been reported to be increasing over the past several years [10]. In humans, E. chaffeensis is the most commonly reported infection followed by $E$. ewingii, but sporadic infections with $E$. muris eauclarensis and "Panola Mountain Ehrlichia" have also been reported $[11,12]$. Changes in the number of Centers for Disease Control and Prevention (CDC)-reported confirmed and probable human ehrlichiosis cases [13] may be associated with the northward expansion of $A$. americanum and $E$. chaffeensis from their historical range in the southern USA [14], with newly established counties reported as far north as South Dakota and New Hampshire [15]. Trends for the other ehrlichiosis agents in humans and dogs are not known. Rhipicephalus sanguineus is found worldwide [16], but different lineages, temperate and tropical, have different geographical distributions [17, 18]. The tropical lineage is associated with outbreaks of Rocky Mountain spotted fever in Mexico [19], but until recently has not been known to be present in the USA. In 2018, tropicallineage ticks were deemed to be established along the entire border from San Diego, California to western Arizona, and as far north as Los Angeles, California [18]. Whether this will impact the risk of exposure to E. canis in the USA remains to be seen.

Veterinarians often test for the presence of Ehrlichia spp. antibodies during canine annual wellness exams, resulting in a robust database of seroprevalence data that enables longitudinal analysis. The availability of over 30,000,000 test results, aggregated at a county and monthly level for vector-borne pathogen exposure in dogs since 2013, allowed us to evaluate temporal trends in the seroprevalence of Ehrlichia spp. and determine where risk of exposure is increasing or decreasing across the USA. This analysis is intended to enable veterinarians and pet owners to determine which preventative practices are best for their patients and pets.

\section{Methods}

\section{Data}

A total of $31,200,847$ tests were reported across the contiguous USA from January 2013 to November 2019 by IDEXX Laboratories, available from [9]. There were 908,619 positive tests, yielding an overall raw seroprevalence of $2.91 \%$. Results are from the SNAP $^{\circledR} 4 \mathrm{Dx}^{\circledR}$ Plus test (IDEXX Laboratories, Inc. Westbrook, ME) which detects antibodies against Ehrlichia spp. as well as antibodies against Anaplasma spp. and Borrelia burgdorferi and antigen from Dirofilaria immitis. No other testing modalities are included in these data. Released in early 2012, the SNAP ${ }^{\circledR}$ $4 \mathrm{Dx}^{\circledR}$ Plus test includes three antigens for the detection of exposure to Ehrlichia spp.: p28 (E. ewingii) and p30/ p30-1 (E. canis) [20]. There is also evidence of cross-reactivity with E. chaffeensis [21]. This test is used commonly by veterinarians throughout the USA both for annual screening during wellness examinations and for diagnosis of suspected vector-borne illness. Results from both veterinary clinics and IDEXX reference laboratories were collated automatically into a centralized database, from which aggregate data were provided to the investigators at a county and monthly scale. Importantly, the reported county is that of the clinic, and no patient histories are known.

Figure 1 displays the number of seropositive tests from each county divided by the number of tests performed in that county; data are aggregated over the study period. Counties which did not report any tests are shown in white.

\section{Model}

We used the Bayesian spatio-temporal binomial regression model developed in [22] to model these data. Let $y_{s t}, n_{s t}$, and $p_{s t}$ denote the number of seropositive tests, total number of tests, and seroprevalence (respectively) from county $s$ in month $t$. Assume that

$$
y_{s t} \mid n_{s t}, p_{s t} \sim \operatorname{Binomial}\left(n_{s t}, p_{s t}\right)
$$

that is, conditional on $n_{s t}$ and $p_{s t}, y_{s t}$ follows a binomial distribution with $n_{s t}$ trials and a success probability of $p_{s t}$. We modeled the seroprevalence via

$$
g\left(p_{s t}\right)=\eta_{s t}=\delta+\beta_{s} t+\xi_{s t},
$$

where $g^{-1}(\cdot)$ is the logistic link function, $\delta$ is a global intercept parameter, $\beta_{s}$ is the regression coefficient for 
county $s$, and $\xi_{s t}$ is a spatio-temporal random effect for county $s$ in month $t$.

We interpret $\beta_{s}$ as the regional trend at county $s$ (note that the $\beta_{s}^{\prime}$ s vary by county). Positive values of $\beta_{s}$ indicate an increasing regional trend at county $s$, while negative values indicate a decreasing trend. A Gaussian predictive process (GPP) [23] was used to model the $\beta_{s}$ 's, allowing them to change smoothly over the study area. GPPs borrow information over space, so that the regional trend at county $s$ is influenced by trends from a relatively large surrounding. GPPs are determined by a mean function, a covariance function, and a set of spatial knot locations. The specifications used here are identical to those used in [22].

The data present with heavy spatio-temporal dependence. Neglecting to account for this dependence can lead to biased estimation and unreliable inference. The spatio-temporal random effects are included to model this dependence via a vector autoregression with an embedded conditional autoregressive (CAR) structure similar to that found in [24] and [25]. For more on CAR models see [26]. For each month $t$, define the vector $\phi_{\mathbf{t}}=\left(\phi_{1 t}, \phi_{2 t}, \ldots, \phi_{S t}\right)^{\prime}$, where $S$ is the total number of counties. We assume that the $\phi_{\mathbf{t}}$ 's are independent realizations from a CAR model, that is, $\phi_{t}$ follows a multivariate normal distribution with mean 0 and variancecovariance matrix given by $\tau^{2}(\mathbf{D}-\rho \mathbf{W})^{-1}$. Here $\tau^{2}>0$ is a marginal variance parameter, $\rho \in(0,1)$ influences the degree of spatial correlation, $\mathbf{W}$ is the county adjacency matrix (i.e. the $(i, j)$ th entry of $\mathbf{W}$ is 1 if counties $i$ and $j$ share a border and 0 otherwise), and $\mathbf{D}$ is a diagonal matrix whose $i$ th diagonal entry is equal to the number of counties sharing a border with county $i$. Define $\xi_{t}=\left(\xi_{1 t}, \xi_{2 t}, \ldots, \xi_{S t}\right)^{\prime}$ to be the vector of spatio-temporal random effects from month $t$. Our assumed vector autoregression is given by $\xi_{1}=\phi_{1}$ and $\xi_{\mathbf{t}}=\zeta \xi_{\mathbf{t}-1}+\phi_{\mathbf{t}}$ for $t \geq 2$. Here $\zeta \in(-1,1)$ is a temporal correlation parameter.

As the model is Bayesian, fully specifying the model necessitates assigning prior distributions to all unknown parameters. The priors used here are identical to those used in [22]. Markov chain Monte Carlo (MCMC) methods are used to sample the model parameters from the posterior distribution, and estimation and inference are performed using this sample in the typical manner. In particular, the posterior mean estimate of the regional trends, (the $\beta_{s}$ terms) is shown below.

We used the posterior parameter sample to estimate the local trends as follows. For each MCMC parameter sample $g$, and each county $s$, fit the following ordinary least squares model

$$
\eta_{s t}^{(g)}=\alpha_{0 s}^{(g)}+\alpha_{1 s}^{(g)} t+\epsilon_{s t}^{(g)}, \quad t=1, \ldots, T .
$$

Here $\eta_{s t}^{(g)}$ is the value of $\eta_{s t}$ calculated using the values of $\delta, \beta_{s}$, and $\xi_{s t}$ obtained on the $g$ th iteration of the posterior sampling algorithm after convergence, the $\epsilon_{s t}$ are independent and identically distributed normal

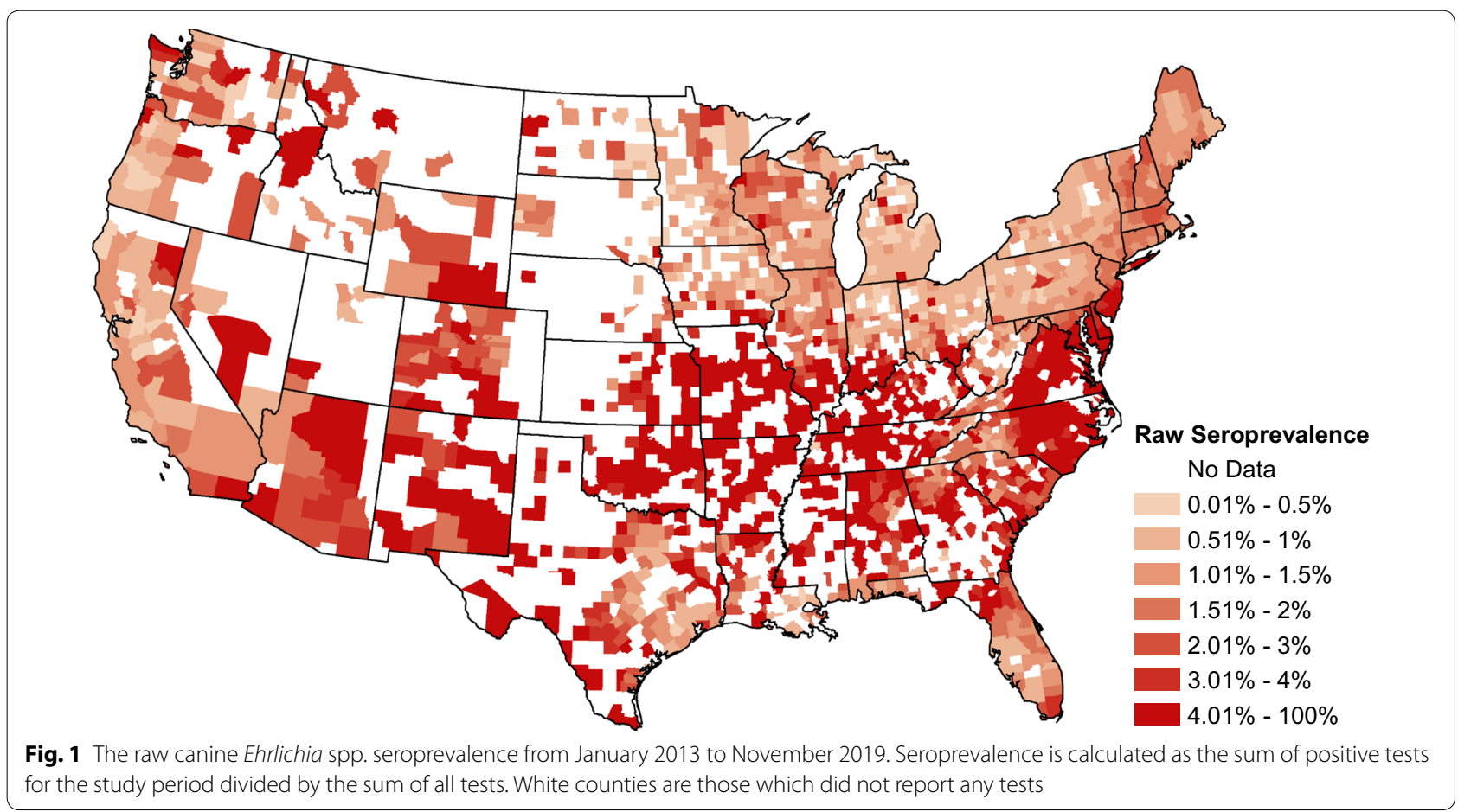


random variables, and $T$ is the total number of months in the study period. For each county $s$, the set of $\widehat{\alpha}_{1 s}^{(g) \text {, s }}$ is a posterior sample of the local trend parameter at county $s$. Estimation and inference for the local trends is conducted using this sample in the usual way. For more information on this model, as well as more details on the calculations of the regional and local trends, see [22].

\section{Assessing model accuracy}

In order to assess the predictive performance of our model, we provide a receiver operating characteristic (ROC) curve. ROC curves plot the sensitivity (the percent of positive tests correctly predicted as positive by the model) against 1 minus the specificity (the percent of negative tests correctly predicted as negative by the model). See $[27,28]$ for more on ROC curves. The area under the ROC curve, or the AUC, is commonly used to assess the predictive performance of models for binary data, with an AUC value greater than 0.7 generally considered indicative of an acceptable model [29].

\section{Results}

\section{Model accuracy}

The ROC curve is shown in Fig. 2. Our model has an AUC of 0.77 , indicating that is has good predictive performance.

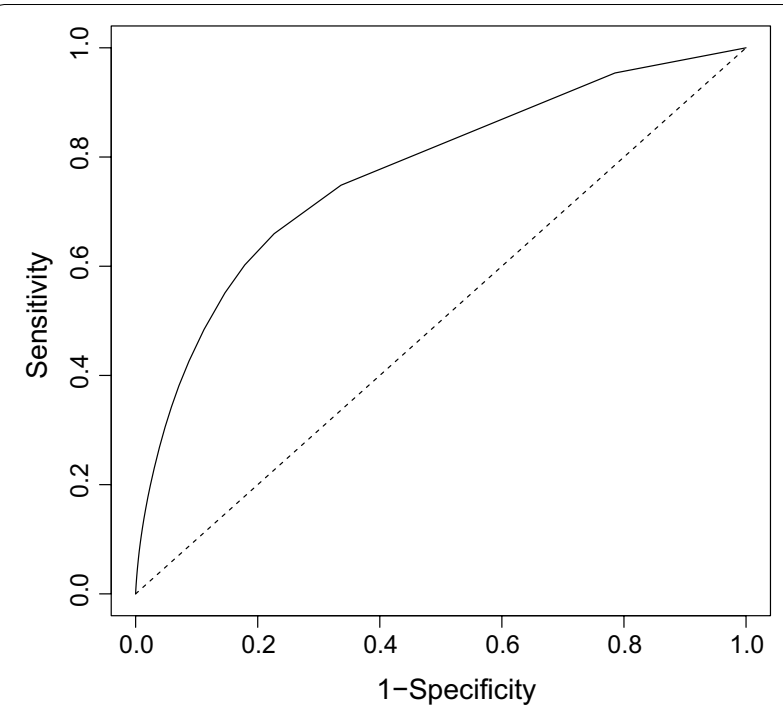

Fig. 2 Receiver operating characteristic (ROC) curve. Plot of the sensitivity (the percent of positive tests correctly predicted as positive by the model) against one minus the specificity (the percent of negative tests correctly predicted as negative by the model)

\section{Regional temporal trends}

This analysis provides two different estimates of the change in Ehrlichia spp. seroprevalence in dogs over the study period. The regional trends provide a largescale estimate of the trend without having to report a fixed national estimate or selecting arbitrary administrative borders for aggregation. Instead, the regional trend estimate for each county was estimated by aggregating data from surrounding counties and allowing near counties to influence the estimate more than those counties farther away. This continuously diminishing influence with increasing distance is formally represented in Fig. 3. For demonstrative purposes only, three areas of high, moderate, and low influence were chosen to represent the distance at which the correlation is above 0.75 (high), between 0.75 and 0.5 (moderate), and below 0.5 (low). The corresponding distances are 0-246 miles $(0-396 \mathrm{~km}), 247-592$ miles $(397-953 \mathrm{~km})$, and $>592$ miles $(>953 \mathrm{~km})$, respectively. The example is extended to the map (Fig. 3b) using Howell County, Missouri as the county of interest.

Regional trends for canine Ehrlichia spp. seroprevalence between January 2013 and November 2019 are shown in Fig. 4a. Displayed are the estimated posterior mean values of the regional temporal trend parameter $\beta_{s}$ from Equation 2. Positive values in red tones indicate regions of increased seroprevalence, while the negative blue tones indicate decreased seroprevalence. Those with a statistically significant increase are shown in Fig. 4b. Significance was assessed with 95\% credible intervals. Any county for which the credible interval of the temporal trend parameter was strictly greater than 0 was deemed to be a region of significant increase.

As shown in Fig. 4, four regional coalescing foci of positive regional trends were present: the Ozarks (Missouri, Arkansas and surrounding states), Virginia and North Carolina, Georgia and southern Texas. The Ozarks region experienced the largest positive trends, followed by Virginia and North Carolina, indicating the two areas of greatest increase. Cross-reactivity between Ehrlichia spp. precludes us from determining which species or vectors are driving these changes. No significant increase in Ehrlichia spp. seroprevalence was observed in the northern or western USA.

\section{Local temporal trends}

In addition to the regional trends, we obtained countylevel trends by extracting $\widehat{\alpha}_{1 s}^{(g)}$ from Equation 3 . The result is shown in Fig. 5a, with statistically significant positive and negative trends as determined by $95 \%$ credible intervals shown in Fig. 5b. The largest clusters of positive trends were present in the western Appalachian region and Missouri and Arkansas. Slightly positive 

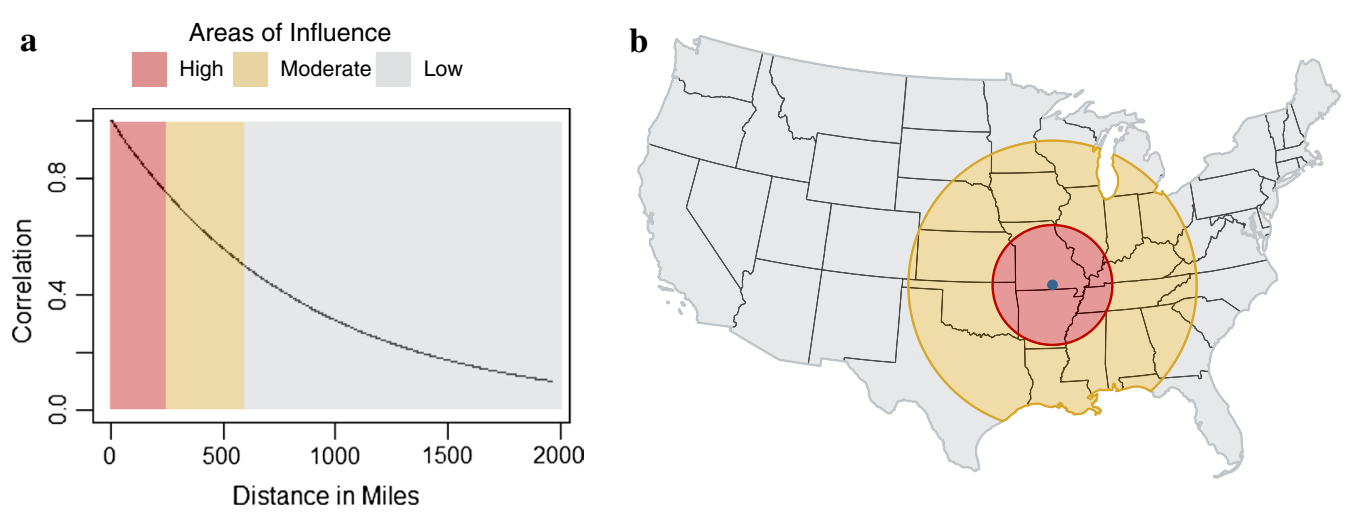

Fig. 3 a The correlation between the trend parameter for canine Ehrlichia spp. for a given county and any other county is determined as a function of distance between those two counties. The curve represents the decaying influence as distance increases between the two counties. This is depicted in panel $\mathbf{b}$ using Howell County, MO as an example. Counties within the red circle have much greater influence over the regional trend estimate for Howell County than those within the grey area

trends extended along the Atlantic coast of the New England states. Other clusters were observed in Illinois, Wisconsin and Minnesota; Florida, and in a few areas of the Mountain states (Fig. 5b). Clusters of negative trends were evident throughout the mid-Atlantic states and New York, particularly North Carolina, Midwest, Texas and California. Counties with both positive and negative trends are scattered throughout the USA, demonstrating the heterogeneity of underlying temporal trends.

\section{Discussion}

The two maps of canine Ehrlichia spp. seroprevalence temporal trends (Figs. 4 and 5) provide (i) a regional perspective that represents the changes that occurred in the population of dogs spanning multiple states, showing the large scale changes in seroprevalence; and (ii) a local perspective that represents changes that occurred within a county-level population of dogs. This local trend provides a more accurate estimation for the change in risk of exposure to the local population of dogs, though neither map can ascribe risk to an individual dog. Because we performed inference on the trends in over 3000 counties, we were faced with the well-known 'multiple testing problem', i.e. the inflation of group type I error rate to levels well above that of each individual test. However, this problem is mitigated through the use of a Bayesian hierarchical model, which has inherent protection against the multiple testing problem [30, 31]. We also show the significance of trends at the $1 \%$ significance level and the standard deviation of the trends in each county to illustrate a more conservative level of significance (Additional file 1: Figures S1, S2).
The interpretation of temporal trends for Ehrlichia spp. seroprevalence in dogs is complicated by the number of cross-reactive species within the genus. The test is labeled to detect E. canis and E. ewingii [32] but has evidence of frequent cross-reactivity with $E$. chaffeensis [21]. Cross-reactivity with other Ehrlichia spp., known or not recognized yet, is also important to consider. Prior studies reported the anomalous presence of Ehrlichia spp. seropositivity in dogs from the upper Midwest [33] that is now believed to be associated with the emergence of the recently recognized E. muris eauclarensis [7]. In addition, "Panola Mountain Ehrlichia" has been detected in dogs [34]. Only the case with $E$. muris reported a weak positive on the SNAP ${ }^{\circledR} 4 \mathrm{Dx}^{\circledR}$ Plus test. Additionally, while these pathogens are transmitted by different tick vectors, the vector ranges have extensive spatial overlap. Ambylomma americanum (vector of E. ewingii and E. chaffeensis) is predominately found in the southern states and along the Atlantic coast into Maine; while $R$. sanguineus (vector of E. canis) and is distributed worldwide [16] with higher numbers occurring in warmer climates [35]. Questing Dermacentor variabilis ticks in the USA have been found to be infected with E. chaffeensis and E. ewingii [36], although prevalence of infection may vary geographically as similar studies in Tennessee and Virginia did not detect Ehrlichia spp. in Dermacentor spp. ticks [37, 38]. The implications of these findings are unknown as only E. canis has been successfully transmitted by $D$. variabilis in an experimental setting [39]. Dermacentor variabilis is widely distributed throughout the USA, but there is no current evidence of range expansion. Its influence on the temporal trends of canine Ehrlichia spp. seroprevalence is unknown. 


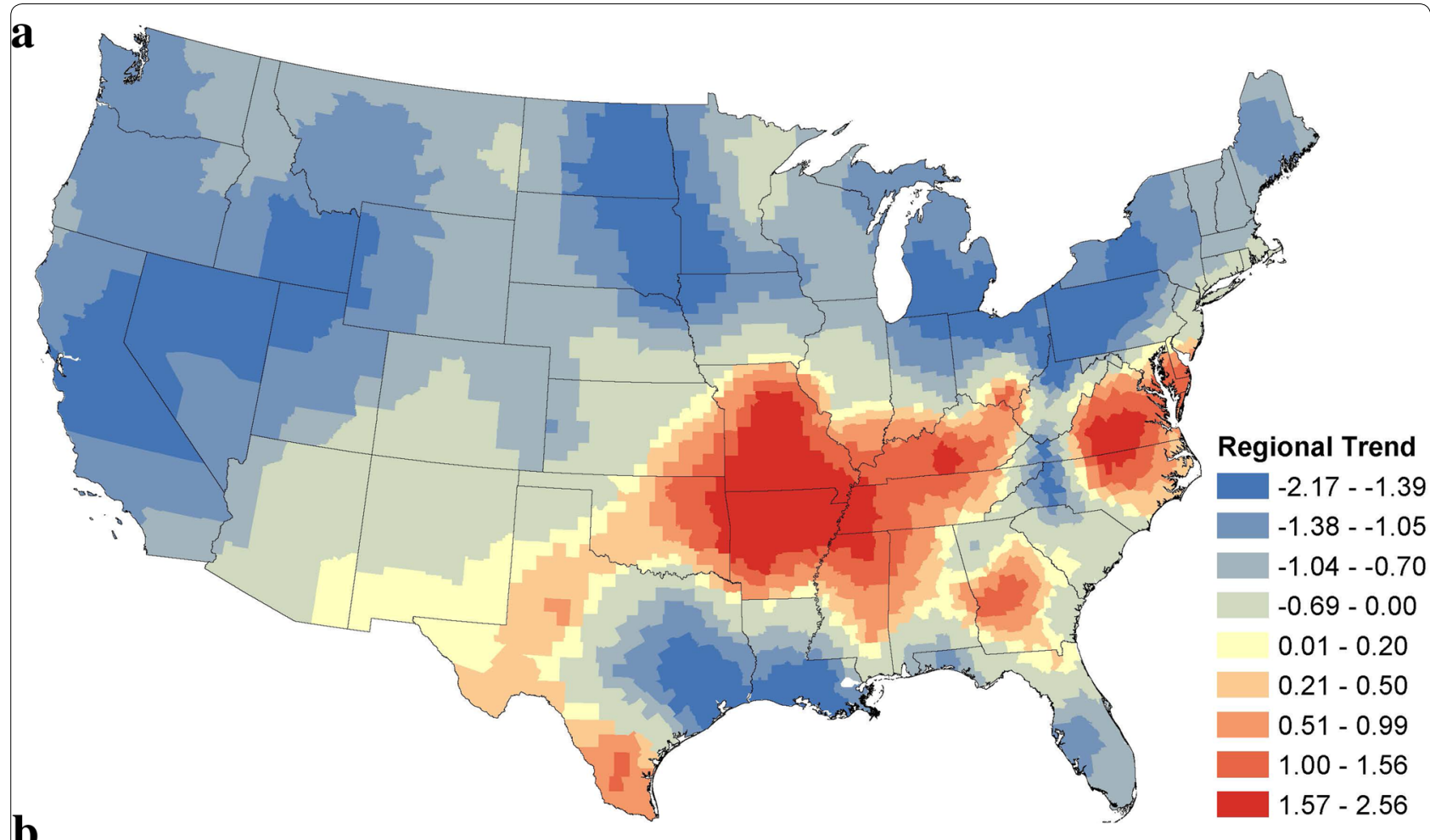

b

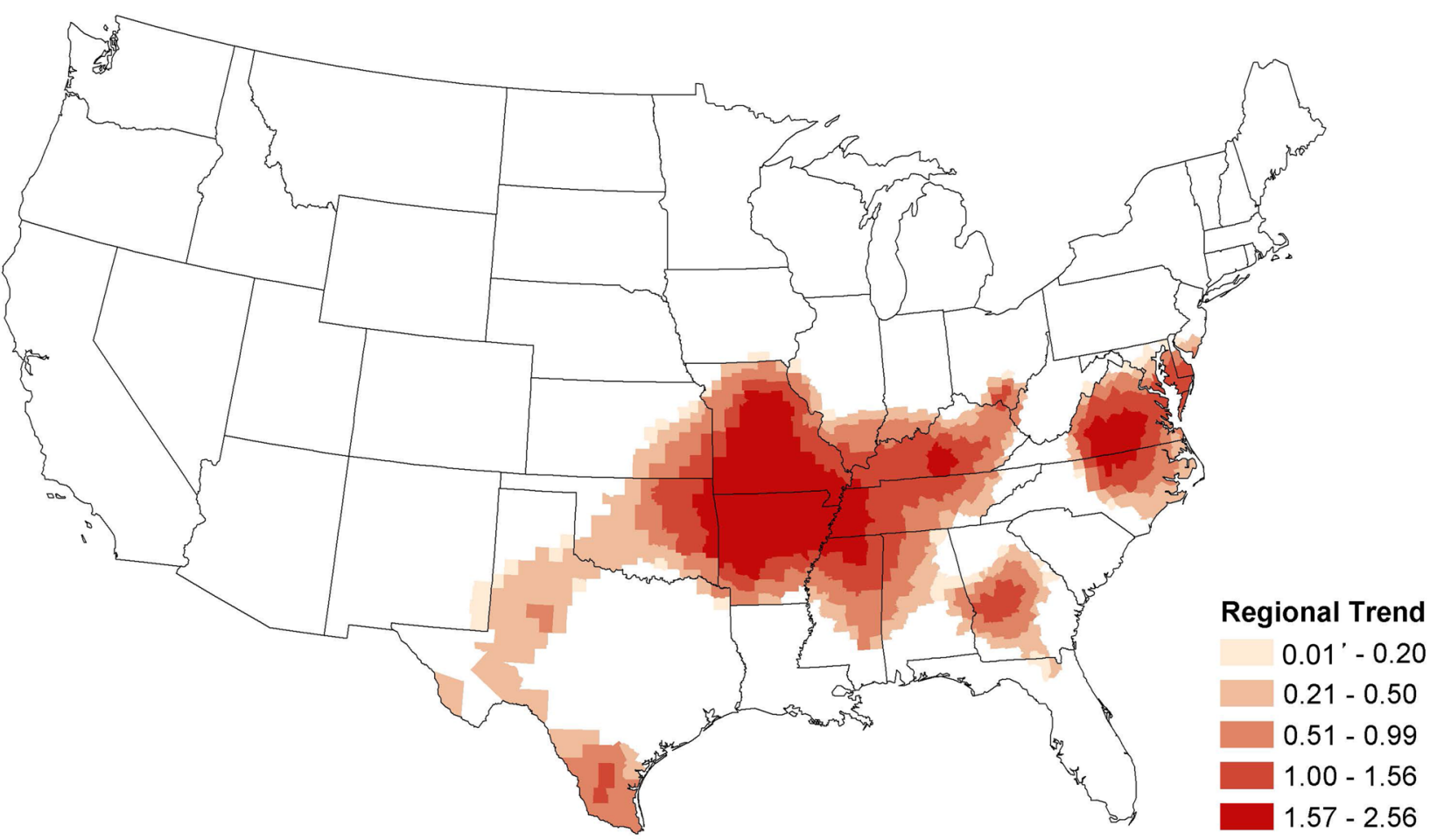

Fig. 4 Posterior mean values of the regional temporal trend parameter for canine Ehrlichia spp. seroprevalence. a Posterior means of the regional temporal trend parameter, $\beta_{5}$ from Equation 2 for all counties. b Counties in red are those for which the $95 \%$ credible interval was strictly positive 


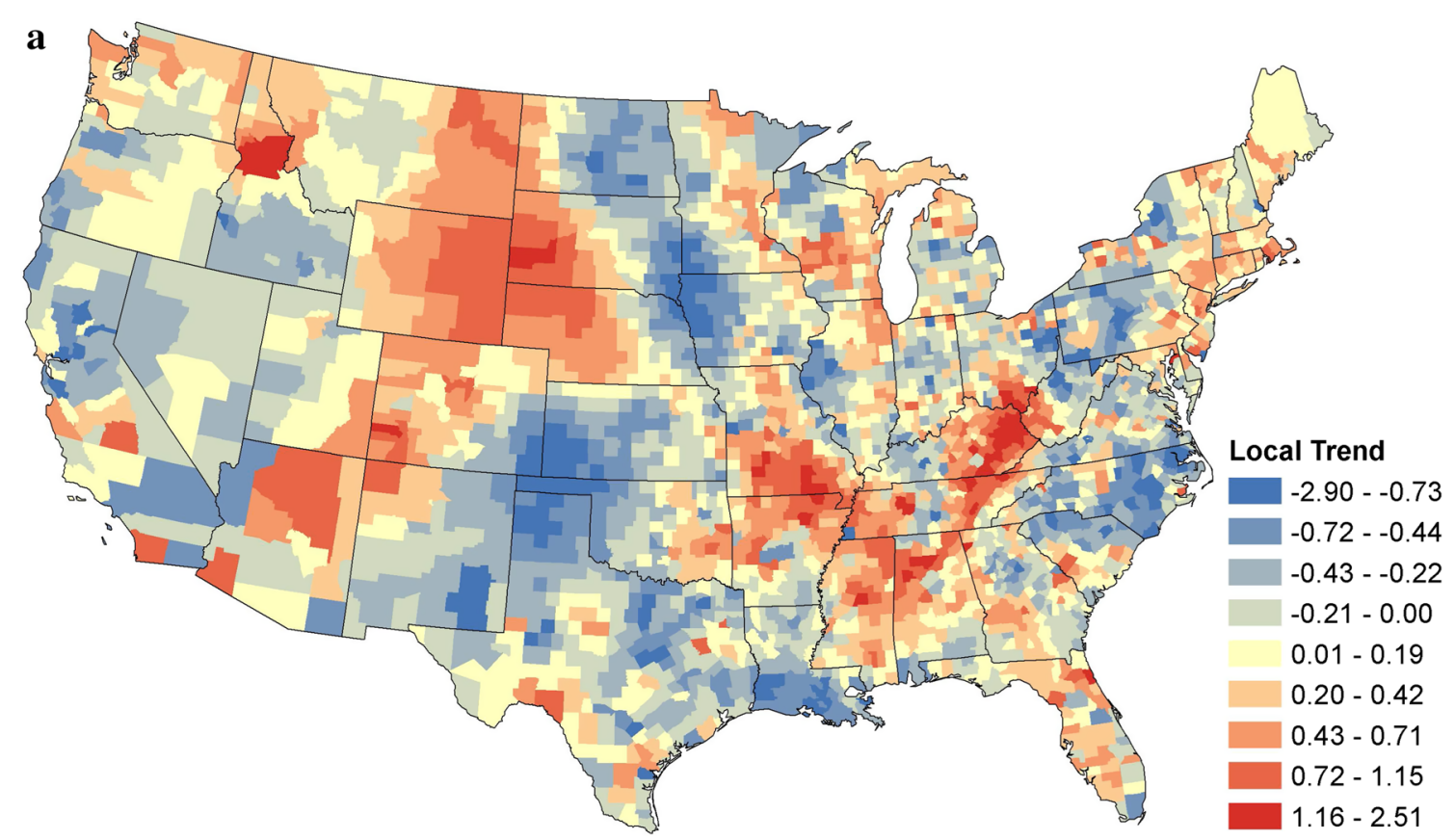

b

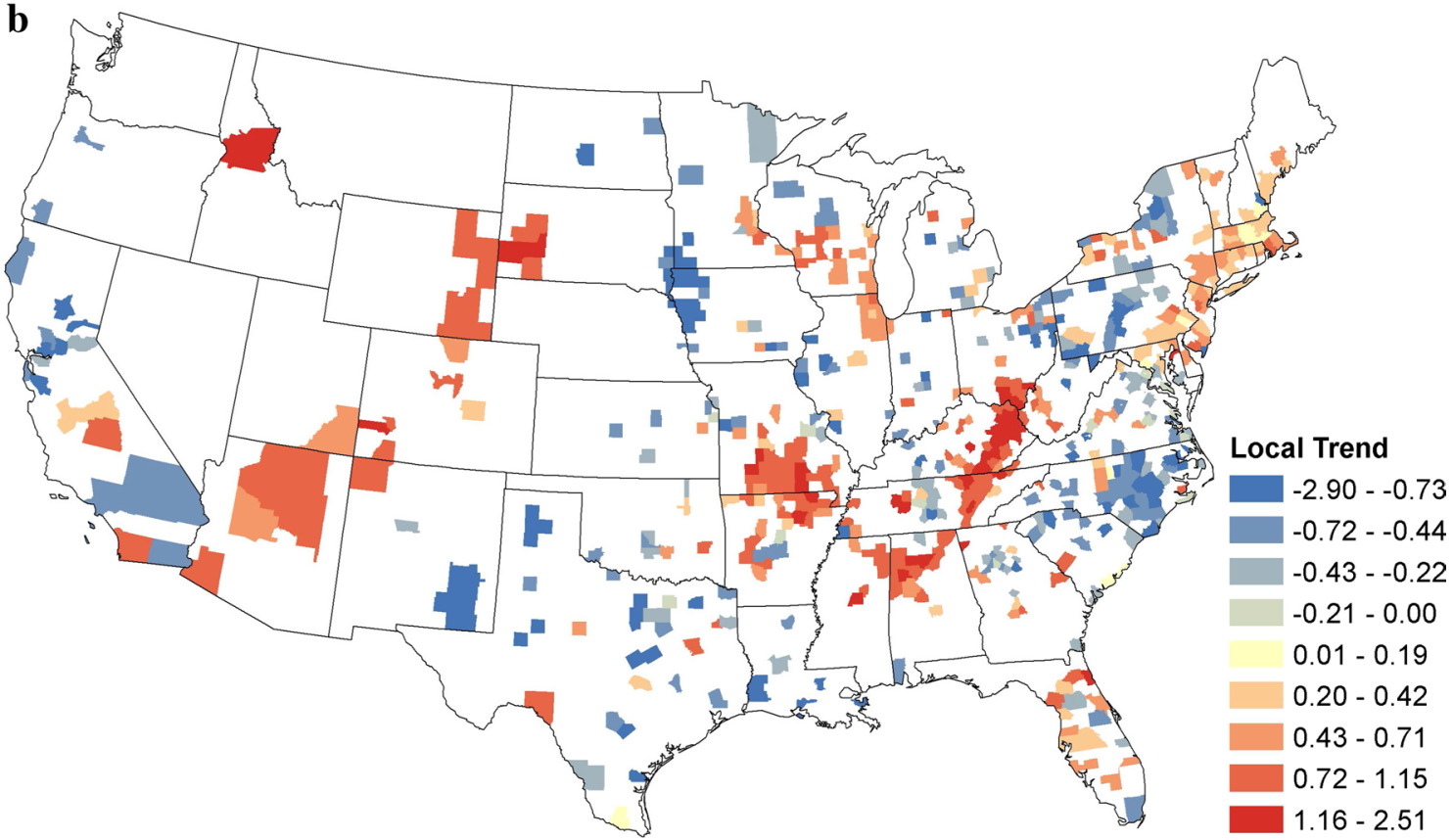

Fig. 5 Posterior mean values of the local temporal trend parameter for canine Ehrlichia spp. seroprevalence. a Posterior means of the local temporal trend parameter for all counties. b Posterior means of the local temporal trend parameter $\alpha_{1 s}$ for counties in which the $95 \%$ credible interval did not contain zero

Prior seroprevalence studies conducted at the species level for canine ehrlichiosis help us to better understand the temporal trends. Among dogs with suspected tick-borne illness in the USA, Qurollo et al. [6] found that E. ewingii was most prevalent with $3.8 \%$ seropositive, followed by E. chaffeensis (3.1\%) and then E. canis
(1.8\%). States with a higher E. canis seroprevalence as compared to either E. ewingii or E. chaffeensis include Alabama, Texas, Colorado and Minnesota (although Minnesota had a small sample size and only one dog tested positive). Another study performed with samples collected for multiple reasons (not necessarily from 
suspected tick-borne disease cases), also found that the seroprevalence of E. canis was higher in Texas compared to the other two pathogens [5]. This might suggest that the regional and local trends we observed in Texas, and possibly Colorado and Alabama, may be driven more by E. canis. In contrast, in both previous studies, the seroprevalence of E. ewingii and E. chaffeensis was higher compared to E. canis in Arkansas and Missouri and many of the surrounding states and in the Atlantic states. Overall, the seroprevalence of $E$. ewingii or E. chaffeensis was 3-30\% higher than E. canis within the same state $[5,38]$. These studies highlight the importance of longitudinal species-specific data to accurately understand the species-specific temporal trends, but data from these two studies do provide insight on which pathogens are likely to influence Ehrlichia spp. trends within a given region.

Although we detected increasing seroprevalence in several regions of the USA, the reasons for these increases cannot be inferred from this study alone and are likely multifactorial and region-specific. However, these results can help researchers build hypotheses and focus future studies in areas experiencing significant changes in order to determine factors associated with temporal trends. Increased prevalence could be associated with higher tick densities, increased prevalence of infected ticks, more frequent interaction with tick habitats, increased wildlife reservoirs or other changes in wildlife population diversity, decreased use of preventative measures, or changes in testing practices among veterinarians. For the pathogens transmitted by $A$. americanum, there is evidence of range expansion for the tick [15] which is believed to be related to, in part, reforestation and subsequent reestablishment of and increases in the white-tailed deer population [40]. The impact of climate change on habitat suitability is expected to allow further northward expansion of A. americanum [41]. Furthermore, risk of exposure is impacted by changes in the interface between developed and undeveloped landscapes, such as occurs in suburban areas. Populations of A. americanum can also be found in planned green spaces within the urban environment [42] and in residential suburban areas [43]. As dogs move with their owners in and out of these areas, their risk of exposure to ticks may change.

Regarding E. canis, there is also evidence of changes in $R$. sanguineus populations. As noted earlier, there are two lineages of this tick species, and evidence of northward range expansion of the tropical lineage has been found in parts of the southern USA, including California [18]. Detection of the tropical lineages have also been reported in Texas, Florida, Illinois and Arizona [44].
Experimentally, ticks from the tropical lineage have been shown to be a more competent vector for $E$. canis, compared to ticks from the temperate lineage [45]. In support of this finding are several studies that detected E. canis in the tropical lineages only [46, 47]. This information is particularly interesting in light of the evidence we present for the increasing trends of $E$. canis prevalence in those states in which the tropical lineage has been detected (Fig. 4).

Factors unrelated to the movement of pathogens and vectors should be considered with interpreting these results. Specifically, the impact of testing practices and preventative practices that reduce the risk of exposure to ticks. Throughout the study period, the number of tests performed within the USA has increased each year across all states [9]. This increase is believed to be associated with the willingness of veterinarians and pet owners to screen dogs for exposure to various vector-borne pathogens during annual wellness visits. As a result, areas with negative trends may be identified because a larger population of healthy and seronegative dogs are being tested over time, resulting in a "dilution" of the seroprevalence. Concurrently, the increase in wellness screening may have been accompanied by an increase in preventative measures, such as the use of acaricides, reducing the risk of exposure, and thus seroprevalence. Alternatively, in areas that do not routinely screen dogs, increased awareness of infection may result in increased testing of ill dogs, thus increasing the seroprevalence. This could reflect a true increase in seroprevalence but could also be an increase in detection of an infection that was already present. While we cannot control for testing and preventative use changes, continuing to monitor temporal changes and interpreting the trends in the context of other known information (e.g. vector range and speciesspecific distribution) will assist in the identification of regions experiencing real and clinically relevant changes in seroprevalence.

The analysis is limited in part by the population of dogs being tested. It is presumed that the majority are under the care of a veterinarian and may be more likely to receive some preventative care, such as acaricides to reduce the risk of tick bites or transmission of pathogens. Thus, the presented results may not represent dogs at higher risk of exposure within the USA (e.g. shelter dogs). Both the regional and local trends should be interpreted within the context of past and current prevalence [9]. The values of the trends in Figs. 4 and 5 are relative to the underlying prevalence. To elaborate, even small changes in areas of very low prevalence may have large trend values, while that same change in high prevalence areas may have a small trend value. Another limitation is the lack of knowledge about individual dogs 
in these data. No histories are known for travel, place of exposure, or reason for testing. Also, some dogs may be tested more than once. However, repeat testing and travel are believed to be a small portion of these data and the focus of the analysis is the temporal trends, which are going to be minimally influenced by these factors. Current practices among many animal shelters and rescues involve the translocation of dogs between different regions of the country. The predominate direction of these movements is from the southern states to the northern states, particularly the Northeast, but animals are also moved from Texas and California to midwestern or western states [48]. The impact of these movements on the prevalence of canine tick-borne disease is unknown at this time, but animals that are exposed in Ehrlichia spp. endemic areas may subsequently test positive in non-endemic areas. Owners and veterinarians should consider this when testing dogs that have been adopted from a shelter or rescue.

Several Ehrlichia spp. are zoonotic, and domestic dogs have been suggested to be effective sentinels for tick-borne pathogens including Borrelia burgdorferi (causative agent of Lyme disease) [49, 50] and Rickettsia rickettsii [51]. However, when interpreting our results, one should keep a few details in mind. Based on data discussed above, we believe that in the Midwest and other parts of the eastern/southeastern USA, much of the Ehrlichia spp. seroreactivity in dogs is due to exposure to E. chaffeensis and E. ewingii $[5,6]$. Most cases of human ehrlichiosis are caused by E. chaffeensis [13], but cases of $E$. ewingii are reported annually in addition to the recently discovered E. muris eauclarensis [12]. The geographical distribution of reported human ehrlichiosis cases [13] closely matches that of canine Ehrlichia spp. seroprevalence [9]. This suggests that dogs in these areas may serve as sentinels for zoonotic ehrlichiae. However, in some regions (e.g. Arizona, Texas), reactivity is likely due to $E$. canis which is not considered a zoonotic pathogen. Future studies are needed to determine how well canine Ehrlichia spp. seroprevalence can estimate the risk of human ehrlichiosis cases and if there are similar spatio-temporal trends for both of these hosts.

\section{Conclusions}

The study presented here highlights the regions of greatest concern for changing canine ehrlichiosis risks in the USA. Given the widespread distribution of the multiple vectors and species of Ehrlichia, nearly all dogs are at risk of exposure, but risk of infection is greatest in the Southeast and Midwest, particularly Missouri and Arkansas, and these regions are also the areas in which we report the largest increasing trends. Pet owners and veterinarians should always practice appropriate care in preventing exposure to ticks (e.g. tick preventatives, thorough examination for ticks, and avoiding tick habitat if possible), especially in areas with high risk.

\section{Supplementary information}

Supplementary information accompanies this paper at https://doi. org/10.1186/s13071-020-04022-4.

Additional file 1: Figure S1. a Posterior mean values of the regional temporal trend parameter for canine Ehrlichia spp. seroprevalence for counties in which the $99 \%$ credible interval was strictly positive. b The posterior standard deviation of the regional temporal trend parameter, $\beta_{s}$ from Equation 2 for all counties. Figure S2. a Posterior mean values of the local temporal trend parameter $\alpha_{1 s}$ for canine Ehrlichia spp. seroprevalence for counties in which the $99 \%$ credible interval did not contain zero. b Posterior standard deviation of the local temporal trend parameter for all counties.

\section{Abbreviations}

AUC: area under the curve; CAR: conditional autoregressive; CDC: Centers for Disease Control and Prevention; GPP: Gaussian predictive process; MCMC: Markov chain Monte Carlo; ROC: receiver operating characteristic.

\section{Acknowledgements}

With much gratitude, we thank IDEXX Laboratories Inc. for providing access to these data which allows studies like this to be conducted.

\section{Authors' contributions}

SCWS, CSM and DAB conceptualized and developed the model; SCWS wrote the methods. JRG, SKN and MJY contributed to the interpretation of the results and writing of the manuscript. JRG, SCWS, CSM, DAB, SKN and MJY reviewed and edited. All authors read and approved the final manuscript.

\section{Funding}

All the authors would like to thank and acknowledge their funding. DAB was funded by the National Science Foundation (NSF) grants CMMI-1563435, EEC1744497, and OIA-1826715. CSM was supported by NSF grant OIA-1826715, National Institutes of Health grant Al121351, and Department of Defense's Office of Naval Research grant N00014-19-1-2295. JRG and SCWS were funded by the Companion Animal Parasite Council (CAPC). The funders had no role in any part of the study or manuscript.

\section{Availability of data and materials}

The dataset analyzed during the present study are available from http://www. capcvet.org.

\section{Ethics approval and consent to participate}

Not applicable (retrospective study using data that are publicly available).

\section{Consent for publication}

Not applicable.

\section{Competing interests}

The authors declare that they have no competing interests.

\section{Author details}

1 Southeastern Cooperative Wild life Disease Study, University of Georgia, Athens 30602, USA. ${ }^{2}$ Arnold School of Public Health, University of South Carolina, Columbia 29208, USA. ${ }^{3}$ School of Mathematical and Statistical Sciences, Clemson University, Clemson 29634, USA. ${ }^{4}$ Comparative Medicine Institute, North Carolina State University College of Veterinary Medicine, Raleigh 27607, USA. ${ }^{5}$ Warnell School of Forestry and Natural Resources, University of Georgia, Athens 30602, USA. 
Received: 14 October 2019 Accepted: 16 March 2020

Published online: 30 March 2020

\section{References}

1. Little SE. Ehrlichiosis and anaplasmosis in dogs and cats. Vet Clin NA Small Anim Pract. 2010;40:1121-40.

2. Anziani OS, Ewing SA, Barker RW. Experimental transmission of a granulocytic form of the tribe Ehrlichieae by Dermacentor variabilis and Amblyomma americanum to dogs. Am J Vet Res. 1990;51:929-31.

3. Anderson BE, Sims KG, Olson JG, Childs JE, Piesman JF, Happ CM, et al. Amblyomma americanum: a potential vector of human ehrlichiosis. Am J Trop Med Hyg. 1993;49:239-44.

4. Groves MG, Dennis GL, Amyx HL, Huxsoll DL. Transmission of Ehrlichia canis to dogs by ticks (Rhipicephalus sanguineus). Am J Vet Res. 1975:36:937-40.

5. Beall MJ, Alleman AR, Breitschwerdt EB, Cohn LA, Couto CG, Dryden MW, et al. Seroprevalence of Ehrlichia canis, Ehrlichia chaffeensis and Ehrlichia ewingii in dogs in North America. Parasit Vectors. 2012;5:29.

6. Qurollo BA, Chandrashekar R, Hegarty BC, Beall MJ, Stillman BA, Liu J, et al. A serological survey of tick-borne pathogens in dogs in North America and the Caribbean as assessed by Anaplasma phagocytophilum, A. platys, Ehrlichia canis, E. chaffeensis, E. ewingii, and Borrelia burgdorferi speciesspecific peptides. Infect Ecol Epidemiol. 2014;3:1-12.

7. Hegarty BC, Maggi RG, Koskinen P, Beall MJ, Eberts MD, Chandrashekar R, et al. Ehrlichia muris infection in a dog from Minnesota. J Vet Intern Med. 2012:26:1217-20.

8. Loftis AD, Mixson TR, Stromdahl EY, Yabsley MJ, Garrison LE, Williamson PC, et al. Geographic distribution and genetic diversity of the Ehrlichia sp. from Panola Mountain in Amblyomma americanum. BMC Infect Dis. 2008;8:54.

9. Companion Animal Parasite Council. Canine ehrlichiosis prevalence maps. https://capcvet.org/maps/2019/all/ehrlichiosis/dog/united-states/. Accessed 1 Apr 2019.

10. Rosenberg R, Lindsey NP, Fischer M, Gregory CJ, Hinckley AF, Mead PS, et al. Vital signs: trends in reported vectorborne disease casesUnited States and Territories, 2004-2016. Morb Mortal Wkly Rep. 2018;67:496-501.

11. Reeves WK, Loftis AD, Nicholson WL, Czarkowski AG. The first report of human illness associated with the Panola Mountain Ehrlichia species: a case report. J Med Case Rep. 2008;2:139.

12. Pritt BS, Sloan LM, Johnson DKH, Munderloh UG, Paskewitz SM, Mcelroy $\mathrm{KM}$, et al. Emergence of a new pathogenic Ehrlichia species, Wisconsin and Minnesota, 2009. N Engl J Med. 2011;365:422-9.

13. Heitman N, Scott Dahlgren F, Drexler NA, Massung RF, Behravesh CB. Increasing incidence of ehrlichiosis in the United States: a summary of national surveillance of Ehrlichia chaffeensis and Ehrlichia ewingii infections in the United States, 2008-2012. Am J Trop Med Hyg. 2016:94:52-60.

14. Stafford KC III, Molaei G, Little EAH, Paddock CD, Karpathy SE, Labonte AM. Distribution and establishment of the lone star tick in Connecticut and implications for range expansion and public health. J Med Entomol. 2018;55:1561-8.

15. Springer $Y P$, Eisen L, Beati L, James AM, Eisen RJ. Spatial distribution of counties in the continental United States with records of occurrence of Amblyomma americanum (Ixodida: Ixodidae). J Med Entomol. 2014;51:342-51.

16. Dantas-Torres F. Biology and ecology of the brown dog tick. Rhipicephalus sanguineus. Parasit Vectors. 2010;3:26.

17. Dantas-Torres F, Latrofa MS, Annoscia G, Giannelli A, Parisi A, Otranto D. Morphological and genetic diversity of Rhipicephalus sanguineus sensu lato from the New and Old Worlds. Parasit Vectors. 2013;6:213.

18. Villarreal Z, Stephenson N, Foley J. Possible northward introgression of a tropical lineage of Rhipicephalus sanguineus ticks at a site of emerging Rocky Mountain spotted fever. J Parasitol. 2018;104:240-5.

19. Eremeeva ME, Zambrano ML, Anaya L, Beati L, Karpathy SE, Santos-Silva MM, et al. Rickettsia rickettsii in Rhipicephalus ticks, Mexicali. Mexico. J Med Entomol. 2011;48:418-21.

20. Stillman BA, Monn M, Liu J, Thatcher B, Foster P, Andrews B, et al. Performance of a commercially available in-clinic ELISA for detection of antibodies against Anaplasma phagocytophilum, Anaplasma platys, Borrelia burgdorferi, Ehrlichia canis, and Ehrlichia ewingii and Dirofilaria immitis antigen in dogs. J Am Vet Med Assoc. 2014;245:80-6.

21. Liu J, Drexel J, Andrews B, Eberts M, Breitschwerdt E, Chandrashekar R. Comparative evaluation of 2 in-clinic assays for vector-borne disease testing in dogs. Top Companion Anim Med. 2018;33:114-8.

22. Self SCW, MCMahan CS, Brown DA, Lund RB, Gettings JR, Yabsley MJ. A large-scale spatio-temporal binomial regression model for estimating seroprevalence trends. Environmetrics. 2018;29:e2538.

23. Banerjee S, Gelfand AE, Finley AO, Sang H. Gaussian predictive process models for large spatial data sets. J R Stat Soc Ser B Stat Methodol. 2008:70:825-48.

24. Rushworth A, Lee D, Mitchell R. A spatio-temporal model for estimating the long-term effects of air pollution on respiratory hospital admissions in Greater London. Spat Spatiotemporal Epidemiol. 2014;10:29-38.

25. Lee $\mathrm{D}$, Lawson A. Cluster detection and risk estimation for spatio-temporal health data. arXiv Prepr arXiv14081191. 2014.

26. Banerjee S, Carlin BP, Gelfand AE. Hierarchical modeling and analysis for spatial data. 2nd ed. Boca Raton: Chapman and Hall/CRC; 2014.

27. Bradley AP. The use of the area under the ROC curve in the evaluation of machine learning algorithms. Pattern Recognit. 1997;30:1145-59.

28. Fawcett T. An introduction to ROC analysis. Pattern Recognit Lett. 2006:27:861-74.

29. Mandrekar JN. Receiver operating characteristic curve in diagnostic test assessment. J Thorac Oncol. 2010:5:1315-6.

30. Scott JG, Berger JO. An exploration of aspects of Bayesian multiple testing. J Stat Plan Inference. 2006:136:2144-62.

31. Gelman A, Hill J, Yajima M. Why we (usually) don't have to worry about multiple comparisons. J Res Educ Eff. 2012;5:189-211.

32. IDEXX Laboratories. SNAP 4Dx Resources and Product Insert; 2018, p 1. https://www.idexx.com/en/veterinary/support/documents-resources/ snap-4dx-plus-test-resources/. Accessed 27 Mar 2019.

33. Bowman DD, Little SE, Lorentzen L, Shields J, Sullivan MP, Carlin EP. Prevalence and geographic distribution of Dirofilaria immitis, Borrelia burgdorferi, Ehrlichia canis, and Anaplasma phagocytophilum in dogs in the United States: results of a national clinic-based serologic survey. Vet Parasitol. 2009;160:138-48.

34. Qurollo BA, Davenport AC, Sherbert BM, Grindem CB, Birkenheuer AJ, Breitschwerdt EB. Infection with Panola Mountain Ehrlichia sp. in a dog with atypical lymphocytes and clonal T-cell expansion. J Vet Intern Med. 2013;27:1251-5.

35. Sykes JE. Ehrlichiosis. In: Sykes JE, editor. Canine and feline infectious diseases. 1st ed. St. Louis: Elsevier Health Sciences; 2014. p. 278-89.

36. Steiert JG, Gilfoy F. Infection rates of Amblyomma americanum and Dermacentor variabilis by Ehrlichia chaffeensis and Ehrlichia ewingii in southwest Missouri. Vector Borne Zoonotic Dis. 2002;2:53-60.

37. Cohen SB, Yabsley MJ, Freye JD, Dunlap BG, Rowland ME, Huang J, et al. Prevalence of Ehrlichia chaffeensis and Ehrlichia ewingii in ticks from Tennessee. Vector Borne Zoonotic Dis. 2010;10:435-40.

38. Wright CL, Gaff HD, Hynes WL. Prevalence of Ehrlichia chaffeensis and Ehrlichia ewingii in Amblyomma americanum and Dermacentor variabilis collected from southeastern Virginia, 2010-2011. Ticks Tick Borne Dis. 2014:5.978-82

39. Johnson EM, Ewing SA, Barker RW, Fox JC, Crow DW, Kocan KM. Experimental transmission of Ehrlichia canis (Rickettsiales: Ehrlichieae) by Dermacentor variabilis (Acari: Ixodidae). Vet Parasitol. 1998;74:277-88.

40. Paddock CD, Yabsley MJ. Ecological havoc, the rise of white-tailed deer, and the emergence of Amblyomma americanum-associated zoonoses in the United States. In: Childs JE, Mackenzie JS, Richt JA, editors. Wildlife and emerging zoonotic diseases: the biology, circumstances and consequences of cross-species transmission. Berlin-Heidelberg: Springer; 2007. p. 289-324.

41. Springer YP, Jarnevich CS, Barnett DT, Monaghan AJ, Eisen RJ. Modeling the present and future geographic distribution of the lone star tick, Amblyomma americanum (Ixodida: Ixodidae), in the continental United States. Am J Trop Med Hyg. 2015;93:875-90.

42. Blanton LS, Walker DH, Bouyer DH. Rickettsiae and ehrlichiae within a city park: is the urban dweller at risk? Vector-Borne Zoonotic Dis. 2014;14:168-70. 
43. Small MM, Laverty SM, King CB, Brennan RE. Tick species establishment in Oklahoma County, Oklahoma, USA, identified by seasonal sampling in residential and non-residential sites. J Vector Ecol. 2019;44:105-11.

44. Jones EO, Gruntmeir JM, Hamer SA, Little SE. Temperate and tropical lineages of brown dog ticks in North America. Vet Parasitol Reg Stud Reports. 2017;7:58-61.

45. Moraes-Filho J, Krawczak FS, Costa FB, Soares JF, Labruna MB. Comparative evaluation of the vector competence of four South American populations of the Rhipicephalus sanguineus group for the bacterium Ehrlichia canis, the agent of canine monocytic ehrlichiosis. PLoS ONE. 2015;10:e0139386.

46. Venzal JM, Estrada-Peña A, Castro O, De Souza CG, Portillo A, Oteo JA. Study on seasonal activity in dogs and ehrlichial infection in Rhipicephalus sanguineus (Latreille, 1806) (Acari: Ixodidae) from southern Uruguay. Parasitol Latinoam. 2007;62:23-6.

47. Cicuttin GL, Tarragona EL, De Salvo MN, Mangold AJ, Nava S. Infection with Ehrlichia canis and Anaplasma platys (Rickettsiales: Anaplasmataceae) in two lineages of Rhipicephalus sanguineus sensu lato (Acari: Ixodidae) from Argentina. Ticks Tick Borne Dis. 2015;6:724-9.
48. American Society for the Prevention of Cruelty to Animals. Animal Relocation; 2018. https://www.aspca.org/animal-placement/animal-reloc ation. Accessed 24 Sept 2019.

49. Mead PS, Goel R, Kugeler KJ. Canine serology as adjunct to human Lyme disease surveillance. Emerg Infect Dis. 2011;17:1710-2.

50. Liu Y, Nordone SK, Yabsley MJ, Lund RB, McMahan CS, Gettings JR. Quantifying the relationship between human Lyme disease and Borrelia burgdorferi exposure in domestic dogs. Geospat Health. 2019;14:750.

51. Elchos BN, Goddard J. Implications of presumptive fatal Rocky Mountain spotted fever in two dogs and their owner. J Am Vet Med Assoc. 2003;223:1450-2

\section{Publisher's Note}

Springer Nature remains neutral with regard to jurisdictional claims in published maps and institutional affiliations.
Ready to submit your research? Choose BMC and benefit from:

- fast, convenient online submission

- thorough peer review by experienced researchers in your field

- rapid publication on acceptance

- support for research data, including large and complex data types

- gold Open Access which fosters wider collaboration and increased citations

- maximum visibility for your research: over $100 \mathrm{M}$ website views per year

At BMC, research is always in progress.

Learn more biomedcentral.com/submissions 